\title{
sEMG-based estimation of human stiffness: towards impedance-controlled rehabilitation
}

\author{
Claudio Castellini, Albert Arquer and Jordi Artigas
}

\begin{abstract}
In rehabilitation robotics, surface electromyography (sEMG) is extensively used as a human-machine interface, mainly for prosthetic/orthotic control purposes. The technique has been proved to be a highly accurate way of detecting a human subject's intended position, force and torque configurations. Widely applied in the clinics, it is gaining even more momentum as polyarticulated, ever-more dexterous selfpowered rehabilitation artifacts appear on the market.

In this paper we present a preliminary result about the usage of the same technique to estimate a human subject's hand stiffness in the presence of force-feedback. A novel force feedback control concept based on the modulation of the robot arm stiffness according to the estimated hand stiffness is presented. Thus, the robot arm is set to mimic the stiffness properties of the subject that is controlling the arm, in real time. Six intact subjects were immersed in a simple teleoperation task, in which force feedback was present; the hand stiffness was measured via force perturbation at the master's manipulandum and associated with the sEMG signals. This live estimation of stiffness was then used to control the impedance of the slave. Experimental results show that this system leads to high positional precision but high contact forces when the estimated stiffness is high, and vice-versa.

The system has potential applications in impedance control of rehabilitation devices such as, e.g., upper / lower limb prostheses, self-powered orthoses and exoskeleta, leading to an ever-better integration with patients.
\end{abstract}

\section{INTRODUCTION}

Surface electromyography (sEMG) is so far probably the most successful human-machine interface for "natural" control of rehabilitation artifacts. sEMG electrodes can be easily applied on a human subject's skin essentially without preparation, and are able to detect the level of muscular activation (motor unit activation potentials), leading to an estimate of the intended positions / forces / torques required at a certain limb [1], [2], [3]. In its simplest form, this signal is used in the clinics since decades to proportionally control one-degree-of-freedom prosthetic grippers; more recently, machine learning has been applied to it, revealing that it can be related with high accuracy to, e.g., the grasping force of a hand [4], the voluntary contraction of single muscles [5] and even to the position of the human arm/hand system [6], [7].

An even more advanced aspect of sEMG is that it can be used to estimate the stiffness of the human limbs. Stiffness plays a crucial role in daily interaction with the environment, especially whenever a rapid switching from high positional accuracy to compliance is required - a task that human

The authors are with the Robotics and Mechatronics Center, DLR German Aerospace Center, D-82234 Oberpfaffenhofen, Germany. email: claudio.castellini@dlr.de beings can naturally accomplish [8], [9]; the estimation of human stiffness using sEMG has been investigated by and large in particular in, e.g., Franklin's work [10], where it was established that the human central nervous system can tune Cartesian stiffness in three dimensions, paving the way to this kind of applications. An sEMG-based estimation of human stiffness is therefore very desirable in order to improve the interaction of a human subject with the environment; force feedback further adds to the realism of the device, and therefore to the feeling of immersion by the patient.

In this paper we present an experiment in this direction. Six intact subjects were immersed in a telepresence scenario and asked to perform a simple reaching task. Their hand stiffness was characterised using torque perturbation (as is standard in literature) and linearly associated to the sEMG signal, denoting the level of muscular activity. In a further test, the impedance of the slave setup was controlled using the sEMG-estimated stiffness, in the presence of force feedback. The results confirm that, when employing high stiffness, the subject was able to pursue the required trajectory with high positional precision, but generated high contact torques when interacting with the environment, and viceversa.

Since telepresence, an enhanced form of teleoperation, is a promising testbed for rehabilitation devices [11], [12], [13], our experiment paves the way for naturally impedancecontrolled prostheses and exoskeleta, with the aim of providing the disabled with an ever-better feeling of immersion in the environment and ownership of such devices.

\section{Telepresence}

Figure 1 is a conceptual sketch of telepresence with stiffness control and force feedback. Telepresence is "the feeling of being present somewhere else": in the ideal case, the telepresence system is modelled as an infinitely stiff, weightless tool [14] connecting the master to the remote environment (upper panel of the Figure) in the most transparent way.

In this work we rather see the tool as a cooperative extension of a (missing) human limb, whose stiffness is modulated upon the subject's desire via sEMG (lower panels of the Figure). Human beings use low stiffness when the environment is unknown (e.g., when moving in the dark) in order to minimise the probability of damage, while they stiffen up when requiring high precision, for instance when putting a thread in a needle. In a rehabilitation scenario, this increases the ease and naturalness of the interaction with unstructured and/or hostile environment; and it constitutes 

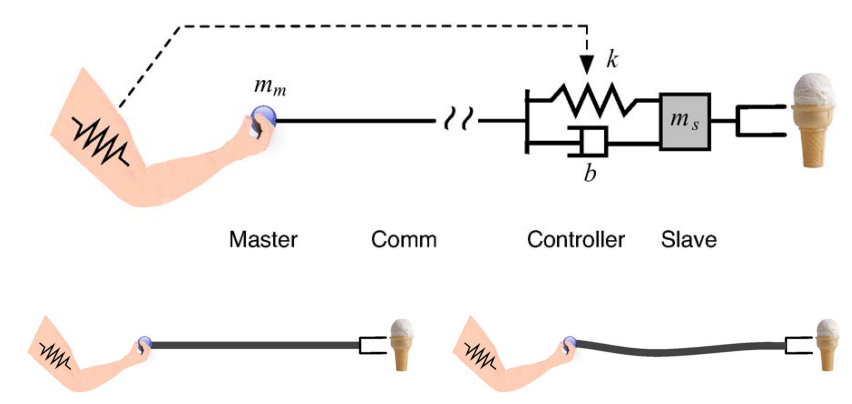

Fig. 1. Conceptual sketch of the telepresence environment. The master is linked to the environment via an ideal tool (upper panel) which can be either rigid (high stiffness, lower left panel) or compliant (low stiffness, lower right panel).

an indication of the intent to move for patient with musculoskeletal conditions such as, e.g., post-stroke or degenerative condition patients.

Similar approaches can be found in the literature. In [15] EMG signals are used to modulate the damping of a bilateral teleoperated controller, in order to cope with time delays, while the Tele-impedance concept [16], [17] is a clear example of successful application of sEMG in this direction, although no force feedback is provided in that case.

\section{SEMG-BASED ESTIMATION OF HAND STIFFNESS}

In this Section we describe the experimental procedure followed in order to estimate the hand stiffness of a pool of human subjects using their sEMG. First the stiffness is characterised, then it is associated to the sEMG.

The approach we follow is a variation of the procedure designed, e.g., in [18] to estimate human impedance, consisting of engaging the subjects in a trajectory-following task while being immersed in an unstable force field, which elicits different levels of stiffness; the stiffness itself is measured using perturbations at the equilibrium point. In our case, the subjects would try and reach a desired angle with a simple onedegree-of-freedom manipulandum, while a graded unstable torque field was applied to the manipulandum itself. Once the target angle was stably reached, a torque perturbation would be issued, the corresponding angular position displacement measured, and the hand stiffness evaluated, as is common, as the ratio between the intensity of the torque perturbation and the angular displacement.

\section{A. Experiment description}

The hardware setup consisted of a one-degree-of-freedom system and ten sEMG electrodes (both described in detail in the Appendix - the manipulandum is visible in Fig. 2, left panel). The sEMG electrodes were placed on the forearm and upper arm according to a loose muscle targeting procedure such as those found, e.g., in [10], [19], [7], although it must be remarked that, with surface EMG, muscle crosstalk cannot be avoided. This is the reason why we choose to use ten electrodes, although the task is monodimensional.
The electrodes were placed as follows: on the forearm, two electrodes above the $M$. Brachioradialis, one above the $M$. Extensor Digitorum Superficialis, two above the M. Flexor Digitorum Superficialis; on the upper arm, one above the M. Biceps Brachii and one above the M. Triceps Brachii; lastly, three electrodes were placed above the M. Deltoideus, one above each Pars of the muscle. (See, e.g., [20] for an overview of the shoulder, upper arm and lower arm muscle anatomy.) A total of 6 able-bodied subjects (age $25.8 \pm$ $1.8 y r s, \min 23, \max 28)$ joined the experiment.

\section{B. Experiment protocol}

Each subject sat comfortably in front of the master setup facing a large PC monitor and was instructed to grab the manipulandum lightly but firmly. The monitor showed a live graphical representation of the manipulandum's position $\theta$ as well as a target angle $\theta^{*}$. The subject was instructed to simply reach the target angle and keep that position until a new target angle would appear (Figure 2, center panel), using the minimum effort required. A tolerance threshold of $\theta_{T}=0.03 \mathrm{rad}$ was allowed when reaching and holding the target angle: the reaching task was considered successful if $\left|\theta-\theta^{*}\right|<\theta_{T}$ for at least one second.

During the task an unstable torque field $\tau=\phi \omega$, proportional to the angular velocity of the manipulandum $\omega$, was continually applied to the manipulandum itself; here $\phi>0$ is a parameter determining the strength of the field, and $\tau$ is the applied torque field. Generally acceptable values of $\phi$ were initially determined to be $[0.05,0.075,0.1,0.125,0.15,0.2,0.25,0.3] \mathrm{Nm} /(\mathrm{rad} / \mathrm{s})$; in a few cases, lower values would be administered according to the subject's preferences and ability. The target angles, $\theta^{*}$, were chosen to be $0 \mathrm{rad}$, corresponding to the manipulandum's vertical position, and $\pm 0.5 \mathrm{rad}$.

Once the task was considered successful, a $0.5 \mathrm{Nm}$ torque step (duration: $t_{p}=300 \mathrm{~ms}$ ) was issued at the manipulandum, at a time $t_{0}$ occurring randomly up to one second after the task completion, that is, after the subject could hold the manipulandum within the tolerance threshold for at least one second.

Each subject was administered a variable number of tasks, for each pair $\left(\theta^{*}, \phi\right)$ in the above specified ranges. The number of repetitions for each pair was variable according to the subject, in the range of 20-50. All in all each experiment lasted about 60' and no subject reported discomfort, fatigue and/or pain. Fig. 2, right panel, shows a typical experiment.

\section{Characterisation of stiffness}

The stiffness was characterised by evaluating the ratio between the amount of the torque perturbation and the angular displacement the manipulandum had undergone within an interval $t_{0}, t_{E}$ :

$$
k_{h}=\frac{\tau\left(t_{E}\right)-\tau\left(t_{0}\right)}{\theta\left(t_{E}\right)-\theta\left(t_{0}\right)}=\frac{\Delta \tau}{\Delta \theta} .
$$

where $t_{E}$, with $t_{p}>t_{E}>t_{0}$, is the time at which the first extremum value of $\theta$ would be found ( $t_{p}$ is the time at which 

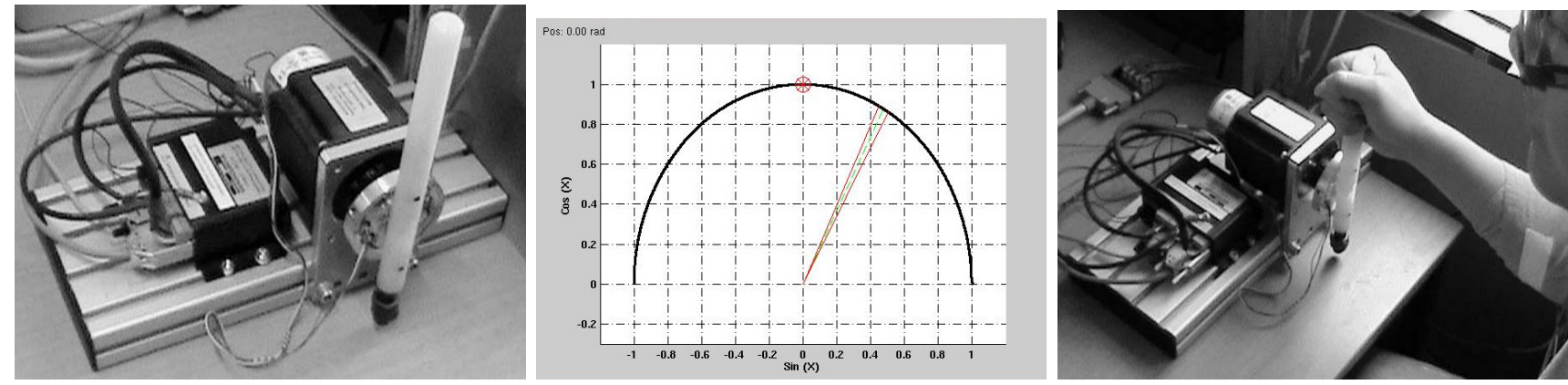

Fig. 2. The experimental setup: (left to right) the one-DoF master setup, the visual stimulus (target angle and current angle of the manipulandum), and a bird's eye view of a subject while performing the experiment.

TABLE I

R-SQUARED COEFFICIENTS OF THE LINEAR FITS OF $k_{h}$ AND $a$ (EQS. 2,3), AND ROOT-MEAN-SQUARE ERROR OF THE MEASURED VS SEMG-ESTIMATED $k_{h}$ (EQ. 4 ), FOR EACH SUBJECT.

\begin{tabular}{|r||rrrrrr|r|}
\hline & $\# 1$ & $\mathrm{~s} \# 2$ & $\mathrm{s \# 3}$ & $\mathrm{s} 4$ & $\mathrm{~s}$ 5 & $\mathrm{s} \# 6$ & mean \pm std. \\
\hline$R^{2}$ for $k_{h}$ & .962 & .856 & .946 & .871 & .978 & .813 & $.904 \pm .067$ \\
$R^{2}$ for $a$ & .896 & .955 & .938 & .888 & .965 & .850 & $.915 \pm .045$ \\
\hline $\operatorname{rMSE}\left[\frac{N m}{\operatorname{rad}}\right]$ & .460 & .371 & .228 & .128 & .222 & .409 & $.303 \pm .129$ \\
\hline
\end{tabular}

the perturbation was issued). The amount of the perturbation was evaluated using the internal torque sensor, rather than fixing the value at the commanded value of $0.5 \mathrm{Nm}$. (The true values actually matched $0.5 \mathrm{Nm}$ within $\pm 0.01 \mathrm{Nm}$; in the case of one subject the sensor malfunctioned, therefore the commanded value was used.)

\section{Characterisation of muscle activation}

The sEMG values read from the ten electrodes were averaged out over the interval $\left[t_{0}-1 s, t_{0}\right]$, that is just before the perturbation occurred. The values read from the electrodes can be directly used without any features extraction, given the type of signal they return (see the Appendix). As the minimum interval allowed to start the perturbation was one second, we assumed that this would indicate the amount of muscle activation required to hold the target angle, given a certain torque-field strength (value of $\phi$ ). Principal Component Analysis (PCA) was then applied to the ten sEMG values, revealing that the first principal component would retain on average about $71.8 \%$ of the signal variance for all subjects. The PCA matrix was evaluated offline for each subject, considering all the tasks performed in the experiment.

The first principal component represents the main mode of muscular activation required in the task at hand, that is, controlling the hand impedance through muscle stiffening and relaxing. We used this eigenvector to evaluate the muscle activation as a single real value, $a>0$.

\section{E. sEMG-based estimation of hand stiffness}

We first checked whether a linear relationship would hold between the strength of the torque field $\phi$, the hand stiffness $k_{h}$, and the muscle activation $a$. Besides being the simplest possible attempt, indications that such a linear relationship holds, albeit in different experiments with respect to ours, are present in literature, see, e.g., [21] and the references therein. Least-squares regression was employed to find the coefficients $\alpha^{\prime}, \alpha^{\prime \prime}, \beta^{\prime}$ and $\beta^{\prime \prime}$ in the following equations:

$$
\begin{aligned}
& k_{h} \approx \alpha^{\prime} \phi+\beta^{\prime} \\
& a \approx \alpha^{\prime \prime} \phi+\beta^{\prime \prime}
\end{aligned}
$$

The R-squared coefficient was employed to check whether the above approximations would be acceptable. Actually, it turned out that this is the case uniformly for all subjects. The coefficients are reported in Table I for each subject and for $k_{h}$ and $a$. This lets us claim that the hand impedance can be estimated using sEMG via linear regression: in fact, by solving for $\phi$ in Eq. 3 and substituting it into Eq. 2 we obtain that

$$
\overline{k_{h}}=\frac{\alpha^{\prime}}{\alpha^{\prime \prime}} a+\left(\beta^{\prime}-\frac{\alpha^{\prime} \beta^{\prime \prime}}{\alpha^{\prime \prime}}\right) \triangleq \alpha^{\prime \prime \prime} a+\beta^{\prime \prime \prime}
$$

Table I also shows the root-mean-squared error obtained when approximating the measured mean values of $k_{h}$ (see Eq. 1) with the approximation $\overline{k_{h}}$ obtained from Eq. 4. Fig. 3 shows the two linear fits and the sEMG-based estimation of $k_{h}$ for a typical subject.

\section{IMPEDANCE CONTROL OF THE TELEPRESENCE SCENARIO}

To model the telepresence scenario, we chose the positioncomputed torque (PF) architecture, one of the most reported schemes in the literature [22]. In this architecture, the master's velocity is transmitted to the slave, where it becomes the reference input of a proportional-derivative controller computing the torques/forces for the slave itself. These forces are then fed back to the master as haptic feedback. A schema of the system is shown in Figure 4.

The equations governing the dynamics of the system are:

$$
\begin{aligned}
Z_{m}(t) & =m_{m} \ddot{q}(t)^{2}+b_{m} \dot{q}(t), \\
\dot{q}_{m}(t) & =\left(\tau_{h}(t)-\tau_{m}(t)\right) Z_{m}^{-1}(t), \\
\tau_{h}(t) & =\dot{q}_{m}(t) Z_{h}(t) .
\end{aligned}
$$

where $\tau_{h}$ is the human torque, $m_{m}$ and $b_{m}$ are the mass and damping coefficients of the master impedance $Z_{m}$, and $Z_{h}$ 

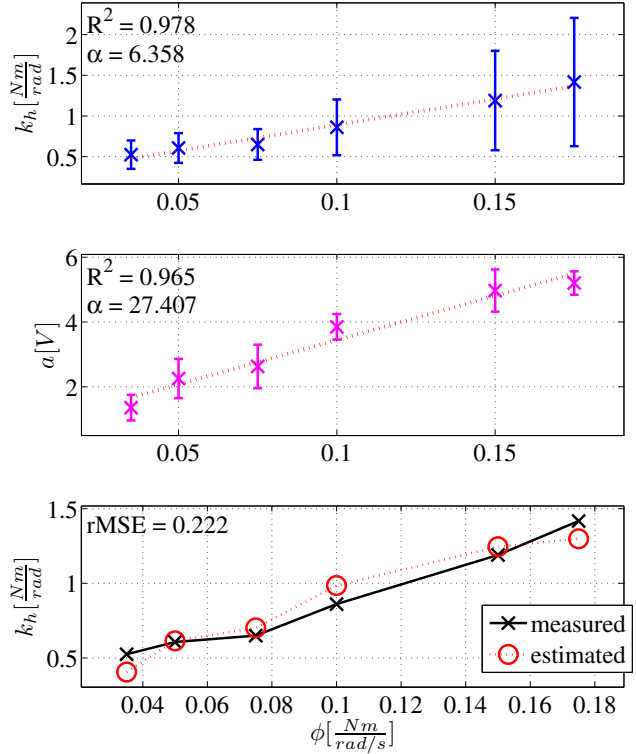

Fig. 3. Linear fits for $k_{h}$ and $a$ according to the strength of the torque field $\phi$, and sEMG-based estimation of $k_{h}$ for a typical subject. (upper panel) Measured values of $k_{h}$ as $\phi$ increases (mean \pm one standard deviation over all tasks for a value of $\phi$ ) and a linear fit; $\alpha$ is the slope of the linear fit. (middle panel) Same as the upper panel, but with measured values of $a$ (muscle activation). (bottom panel) measured and sEMG-estimated values of $k_{h}$ as $\phi$ increases, and related root-mean-square error.

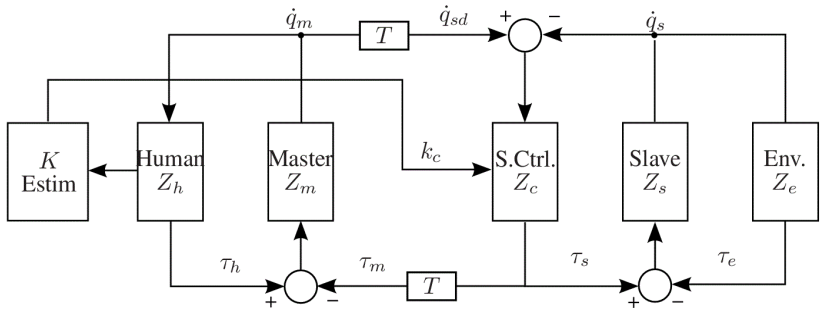

Fig. 4. Block diagram of the PF teleoperation architecture.

is the human impedance. (Similar equations for the slave can be analogously derived.) The sEMG estimation coefficients $\left(\alpha^{\prime \prime \prime}, \beta^{\prime \prime \prime}\right)$ from Eq. 4 , as well as the first eigenvector of the PCA matrix, $v_{E M G}$, are used to compute the desired control stiffness as follows: first, the human stiffness, as estimated using sEMG, is computed,

$$
\overline{k_{h}(t)}=\alpha^{\prime \prime \prime}\left(v_{E M G} \cdot e(t)\right)+\beta^{\prime \prime \prime} \text {. }
$$

where $e$ are the sEMG values, as directly recorded by the electrodes placed on the subject's arm and forearm. This value needs to be normalized within the stability boundaries of the system $K_{p}^{\min }$ and $K_{p}^{\max }$, leading to

$$
k_{c}(t)=\left(K_{p}^{\max }-K_{p}^{\min }\right) \cdot \frac{\overline{k_{h}(t)}-k_{h}^{\min }}{k_{h}^{\text {max }}-k_{h}^{\text {min }}}+K_{p}^{\text {min }} .
$$

where $k_{c}$ is the impedance input to the controller. Using then Equation 7, we obtain $\tau_{s}(t)=b\left(\dot{q}_{s d}(t)-\dot{q}_{s}(t)\right)+k_{c}(t) \int_{0}^{t}\left(\dot{q}_{s d}(t)-\dot{q}_{s}(t)\right) d \tau$

where $\tau_{m}, \dot{q}_{m}$ and $\tau_{s}, \dot{q}_{s}$ are the master and slave forces and velocities and $b$ is the damping coefficient.

\section{Stability}

The stability of the sEMG-based bilateral controller depends on the time delay between the master and the slave, the sampling frequency, the discretization, the master and slave dynamics, the human in the loop, and the physical limitations of the device. Stability analysis depending on these factors has been well studied in the past [23], [14], [24]. Following the literature, for a given delay, damping $b$ and master/slave dynamics, the stability margins for $k_{c}$ can be calculated, using for instance the Nyquist stability criterion:

$$
K_{p}^{\min } \leq k_{c}(t) \leq K_{p}^{\max }, \quad \forall t \geq 0
$$

Figure 5 shows the stability regions for $k_{c}$ as a function of $b$ and the communication time delay between master and slave.

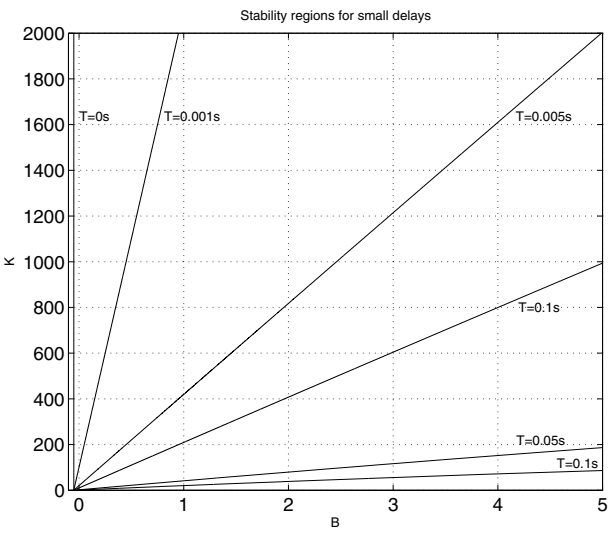

Fig. 5. Stability regions for $\overline{k_{h}}$ as a function of $b$ and the time delay, $T$.

\section{EXPERIMENTAL RESULTS}

In order to validate the approach, a further experiment was deigned and enforced: a series of trajectory-following tasks was performed anew by one of the subjects involved in the characterisation experiment (Section II). The selected subject obtained in this case R-squared coefficients of $k_{h}$ and $a$ of 0.948 and 0.923 and a rMSE of the measured vs. sEMGestimated $k_{h}$ of $0.16 \frac{\mathrm{Nm}}{\mathrm{rad}}$, in the range of the data shown in Table I. The trajectory-following task consisted in driving the manipulandum from 0 to $-\pi / 2$ and then to $\pi / 2$ rads. A rigid object was put in the range of the slave at the positive end of the slave, corresponding to about $\pi / 2$ rads. Position and torques of the manipulandum and the slave were recorded, as well as the estimated stiffness. As a simplified scenario, 
the test subject was asked to alternatively keep all muscles as relaxed as possible (low stiffness) or to slightly contract them (high stiffness). The task was performed 10 times in low stiffness mode and 10 times in high stiffness. In the low stiffness scenario, the estimated average $k_{h}$ was $0.75 \frac{\mathrm{Nm}}{\mathrm{rad}}$. In the high stiffness mode it was $2.51 \frac{\mathrm{Nm}}{\mathrm{rad}}$. (High stiffness is hereby meant as significantly higher than that estimated in the low-stiffness case.) Fig. 6 shows the mean values, standard deviations and single values of the rMSE between the master and slave positions during the tasks, and of the reaction torque recorded during the contact between the slave and the rigid object.
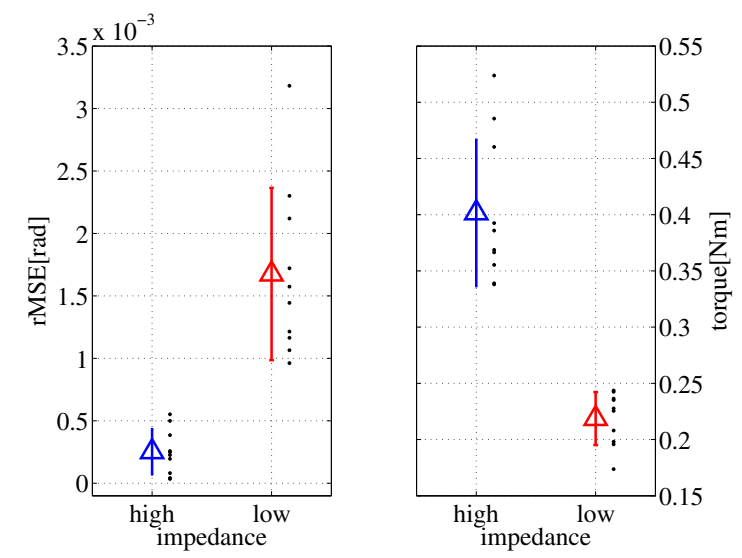

Fig. 6. Mean values \pm one standard deviation (together with single experiment values): rMSE between master and slave position and average torque during the contact with the rigid object, when either low or high stiffness is estimated and applied. The sEMG-estimated $k_{h}$ was $0.75 \frac{\mathrm{Nm}}{\mathrm{rad}}$ in the low-stiffness scenario, and $2.51 \frac{\mathrm{Nm}}{\mathrm{rad}}$ in the high-stiffness scenario.

It is clear from the figures that in the low stiffness mode, the rMSE between the master and slave position is significantly larger than in the high-stiffness mode, while the feedback torque is significantly smaller in the low stiffness mode and vice-versa (in both cases a two-samples t-test was performed, obtaining a $p$-value $\ll 0.01$ ). These results show that high stiffness results in small tracking errors and high contact torques, whereas low stiffness results in high tracking errors but low interaction torques.

Figure 7 and 8 further illustrate this behavior. In the first Figure, the subject makes contact with the rigid object with low and high stiffness while trying to keep the tracking error constant; the first contact results in a low reaction torque, while the second results in a high torque. (In both figures, the sEMG-estimated stiffness $k_{h}$ and that applied at the slave, $k_{c}$, are displayed.) In the second Figure, the subject is asked to try to keep the torque constant during both contacts. According to the expected behavior, the first contact results in high tracking error due to the low impedance. The second minimizes the tracking error due to the high stiffness. Note the stiffness values are saturated, according to $K_{p}^{\max }$.

\section{DISCUSSION AND CONCLUSIONS}

The experimental results shown in this paper prove that a further element to improve the feeling of immersion and
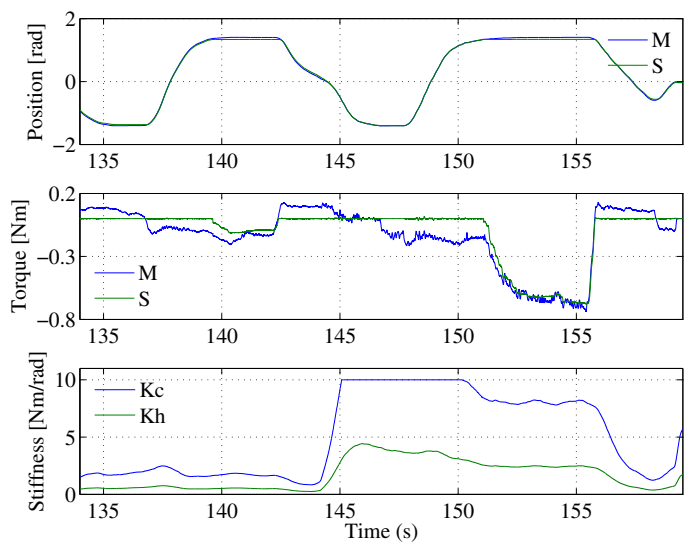

Fig. 7. The subject tries to keep the position error constant during both contacts with different stiffness modes resulting in different reaction torques.
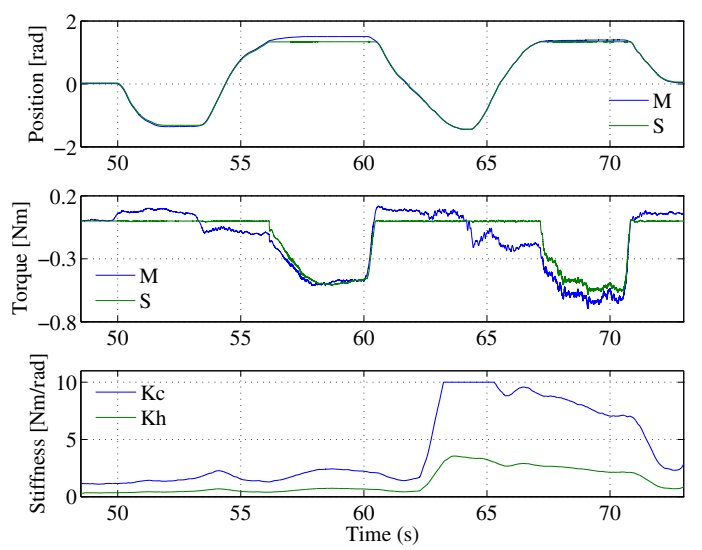

Fig. 8. The subject tries to keep the contact torques constant during both contacts with different stiffness modes resulting in different position error values.

ownership can be added to a telepresence scenario, namely the natural control of the slave impedance, realised via surface electromyography. In our simple one-degree-of-freedom teleoperation task, the hand stiffness of six healthy subjects was characterised and linearly estimated using sEMG with excellent accuracy (R-squared regression coefficients around 0.90). In a further test, the slave's impedance could be controlled in real time using the estimated stiffness, leading to significantly high precision and contact torques when high stiffness was commanded, and vice-versa. With respect to previous approaches, e.g., that shown in [16], [17], we added force feedback to augment the feeling of telepresence and enforce a natural control of robotic impedance by a human subject.

The fact that the first principal component of the sEMG signal explains more than $70 \%$ of the signal variance hints at the fact that there actually is only one main mode of muscular activation during this task. We speculate that this is due to the task being one-dimensional, but it is worth remarking that in a more complex task, say two- or three-dimensional, this could no longer be the case.

This paves the way to intuitive impedance control of 
rehabilitation artifacts by the disabled; one potential application would be, e.g., that of enabling a patient of muscular weakness control not only the intended motion / torques of an arm exoskeleton, but also its stiffness, improving the interaction of such a robotic device with the environment, reducing the risk of damage to the environment itself as well as to human assistants.

Future work will deal with the extension of the approach to multiple degrees of freedom. As the tasks increases in complexity and the workspace grows, the operator's limb dynamics will gain relevance and become non-negligible; the generalization of the exposed techniques will require a reliable model of the operator's arm.

\section{APPENDIX}

\section{A. Experimental Setup}

The master/slave setup was a one-degree-of-freedom system by Sensodrive (see Figure 2). Both master and slave devices are based on a brushless motor with a nominal torque of $0.7 \mathrm{~N} \cdot \mathrm{m}$ and a peak torque of $2 \mathrm{~N} \cdot \mathrm{m}$. The system ran on a real-time OS (QNX) at a frequency of $1 \mathrm{KHz}$ and each device was equiped with a 10-bit torque sensor with a dynamic range of approximately $\pm 1.7 \mathrm{~N} \cdot \mathrm{m}$. Simulink and the Real-Time Workshop were used to enforce the master/slave connection. (During the characterisation phase only the master device was used.) The sEMG signals generated by the electrodes were sampled using a standard ADC card at a rate of $500 \mathrm{~Hz}$, granting a resolution of 11 bits.

\section{B. Surface electromyography}

We used ten Otto Bock MyoBock 13E200 active, doubledifferential sEMG electrodes, which already provide a rectified, filtered signal. The use of this signal rather than the raw one is preferred here since digital processing is considerably simplified. The unavoidable delay of around $200 \mathrm{~ms}$ could be hereby neglected thanks to the chosen data processing. (The effectiveness of these electrodes has been demonstrated many a time in literature, e.g. in [4], [25].)

\section{REFERENCES}

[1] Carlo J. De Luca. Surface electromyography: Detection and recording, 2002. Copyright 2002 by DelSys, Inc.

[2] R. Merletti, A. Botter, C. Cescon, M.A. Minetto, and T.M.M. Vieira. Advances in surface EMG: Recent progress in clinical research applications. Critical reviews in biomedical engineering, 38(4):347-379, 2010.

[3] R. Merletti, A. Botter, A. Troiano, E. Merlo, and M.A. Minetto. Technology and instrumentation for detection and conditioning of the surface electromyographic signal: State of the art. Clinical Biomechanics, 24:122-134, 2009.

[4] Claudio Castellini and Patrick van der Smagt. Surface EMG in advanced hand prosthetics. Biological Cybernetics, 100(1):35-47, 2009.

[5] R. Merletti, M. Aventaggiato, A. Botter, A. Holobar, H. Marateb, and T.M.M. Vieira. Advances in surface EMG: Recent progress in detection and processing techniques. Critical reviews in biomedical engineering, 38(4):305-345, 2011.

[6] P.K. Artemiadis and K.J. Kyriakopoulos. EMG-based control of a robot arm using low-dimensional embeddings. Robotics, IEEE Transactions on, 26(2):393 -398, april 2010.
[7] Jörn Vogel, Claudio Castellini, and Patrick van der Smagt. EMG-based teleoperation and manipulation with the DLR LWR-III. In Proceedings of IROS - International Conference on Intelligent Robots and Systems, pages 672-678, 2011.

[8] F. A. Mussa-Ivaldi, N. Hogan, and E. Bizzi. Neural, mechanical and geometric factors subserving arm posture in humans. J.Neurosci., $5: 2732-2743,1985$.

[9] R. Shadmehr, F. A. Mussa-Ivaldi, and E. Bizzi. Postural force fields of the human arm and their role in generating multi-joint movements. J.Neurosci., 13:45-62, 1993.

[10] D. W. Franklin, G. Liaw, T. E. Milner, R. Osu, E. Burdet, and M. Kawato. Endpoint stiffness of the arm is directionally tuned to instability in the environment. The Journal of Neuroscience, 27(29):7705-77016, 2007.

[11] M. D. Duong, K. Terashima, T. Miyoshi, and T. Okada. Rehabilitation system using teleoperation with force-feedback-based impedance adjustment and EMG-moment model for arm muscle strength assessment. Journal of Robotics and Mechatronics, 22(1):10-20, 2010.

[12] M.H. Rahman, T. K-Ouimet, M. Saad, J.-P. Kenne, and P.S. Archambault. Tele-operation of a robotic exoskeleton for rehabilitation and passive arm movement assistance. In Robotics and Biomimetics (ROBIO), 2011 IEEE International Conference on, pages 443-448, Dec 2011.

[13] X. B. Guo and Y Zhai. Application of teleoperation for rehabilitation training robot. Advanced Materials Research, 267:472—475, 2011.

[14] Yasuyoshi Yokokohji and Tsuneo Yoshikawa. Bilateral Control of Master-Slave Manipulators for Ideal kinesthetic Coupling - Formulation and Experiment. IEEE Transactions on Robotics and Automation, 10(5):605-620, October 1994.

[15] F. Mobasser and K. Hashtrudi-Zaad. Adaptive teleoperation control using online estimate of operator's arm damping. In Decision and Control, 2006 45th IEEE Conference on, pages 2032-2038, Dec.

[16] Arash Ajoudani, Nikolaos G. Tsagarakis, and Antonio Bicchi. Teleimpedance: Towards transferring human impedance regulation skills to robots. In ICRA, pages 382-388, 2012.

[17] Arash Ajoudani, Nikos Tsagarakis, and Antonio Bicchi. Teleimpedance: Teleoperation with impedance regulation using a bodymachine interface. The International Journal of Robotics Research, 31(13):1642-1656, 2012.

[18] E. Burdet, R. Osu, D. W. Franklin, T. E. Milner, and M. Kawato. The central nervous system stabilizes unstable dynamics by learning optimal impedance. Nature, 414:446-449, 2001.

[19] P. K. Artemiadis and K. J. Kyriakopoulos. EMG-based control of a robot arm using low-dimensional embeddings. IEEE Transactions on Robotics, 26(2):393-398, 2009.

[20] M. Schünke, E. Schulte, and U. Schumacher. Prometheus LernAtlas der Anatomie: Allgemeine Anatomie und Bewegungssystem. Thieme, Stuttgart, 2011.

[21] Hannes Höppner, Dominic Lakatos, Holger Urbanek, Claudio Castellini, and Patrick van der Smagt. The grasp perturbator: calibrating human grasp stiffness during a graded force task. In Proceedings of ICRA - International Conference on Robotics and Automation, pages 3312-3316, 2011.

[22] Jordi Artigas, Jee-Hwan Ryu, C. Preusche, and G. Hirzinger. Network representation and passivity of delayed teleoperation systems. In Intelligent Robots and Systems (IROS), 2011 IEEE/RSJ International Conference on, pages 177-183, Sept 2011.

[23] Dongjun Lee and Mark W. Spong. Passive bilateral teleoperation with constant time-delay. IEEE Transactions on Robotics and Automation, 22(2):269-281, April 2006.

[24] Jordi Artigas, Jee-Hwan Ryu, and Carsten Preusche. Position drift compensation in time domain passivity based teleoperation. In IEEE International Conference on Robotics and Intelligent Systems, Taipei, Taiwan, April 2010.

[25] Claudio Castellini, Emanuele Gruppioni, Angelo Davalli, and Giulio Sandini. Fine detection of grasp force and posture by amputees via surface electromyography. Journal of Physiology (Paris), 103(35):255-262, 2009. 